\title{
Microwave Assisted Rapid Bio-based Synthesis of Gold Nanorods Using Pigment Produced by Streptomyces coelicolor klmp33
}

\author{
Deene Manikprabhu and K. Lingappa ${ }^{\dagger}$ \\ Department of Microbiology, Gulbarga University, Gulbarga-585106, Karnataka, India \\ [Manuscript received 14 April 2013, in revised form 8 May 2013] \\ (c) The Chinese Society for Metals and Springer-Verlag Berlin Heidelberg
}

\begin{abstract}
Over past few years various protocols have been developed to produce gold nanorods by chemical synthesis but, most of the chemical used in synthesis are toxic which shows immense effect on environment. In the present investigation, we developed a simple bio-based synthesis of gold nanorods employing blue pigment produced by Streptomyces coelicolor klmp33 using microwave irradiation. The pigment used to reduce $\mathrm{HAuCl}_{4}$ in solution to yield gold nanorods with in $120 \mathrm{~s}$. The gold nanorods were characterized by UV-visible spectroscopy; transmission electron microscopy (TEM), X-ray diffraction (XRD) and Fourier transform infrared spectroscopy (FTIR). The bio-based synthesis developed in this method is safe, eco-friendly, rapid and appropriate way for bulky synthesis of gold nanorods.
\end{abstract}

KEY WORDS: Gold nanorods; Streptomyces coelicolor klmp33; Microwave irradiation

\section{Introduction}

Gold belong to the family of "metal of antiquity", having the history with manhood dating back to 4000 BC. Gold in the form of Swarna bhasma (gold ash) used for medicinal purposes in ancient India ${ }^{[1]}$ and later it is believed that gold nanoparticles was the main reason for the effectiveness of this holy ash ${ }^{[2]}$. Various physical and chemical techniques have been used successfully to produce nanoparticles, but these methods involve the use of hazardous chemicals which pose potential environmental and biological risks ${ }^{[3]}$. To overcome this problem many biological synthesis, using fungus like Neurospora crassa ${ }^{[4]}$, plant extract like Cocculus hirsutus ${ }^{[5]}$ bacteria like Streptomyces griseus $^{[6]}$ used as safe, eco friendly and alternate way to synthesize of gold nanoparticles.

Current interest in nanotechnology has stimulated a number of studies on the use of a variety of shapes of gold nanoparticles in different fields. The morphologies of interest include nanospheres, nanoshells,

† Corresponding author. Prof., Ph.D.; Tel.: +91 9448204513; Fax: +91 8472 263206; E-mail address: lingappak1 @rediffmail.com, lingappak123@gmail.com (K. Lingappa)

DOI: $10.1007 / \mathrm{s} 40195-013-0217-6$ nanocages, and nanorods ${ }^{[7]}$. Among various shapes, gold nanorods are of great importance, having applications in optical filtering, sub wavelength imaging, data storage, sensor devices and also as hyperthermia agents to heat-kill cancer cells, the more rod-like the particle, the greater the heat that it can generate and thus the better it can act as a hyperthermia agent $^{[8,9]}$, but synthesis of gold nanorods is a challenging task. However, many different processes to synthesis gold nanorods have been reported, mainly includes template-type, electrochemical-type, and wet chemistry-type but these techniques remain expensive and some processes include toxic chemical which shows adverse effect on environment ${ }^{[10,11]}$. So, research for simple and non-toxic process for gold nanorods synthesis is required. Accepting this challenging task, we made an attempt to synthesize gold nanorods using blue pigment produced by Streptomyces coelicolor klmp33 by microwave irradiation.

Streptomyces coelicolor is the well known blue pigment producing gram positive bacteria ${ }^{[12]}$. Review of the literature revealed that synthesis of gold nanorods using Streptomyces coelicolor pigment has been unexplored, which aroused our interest in selecting this particular organism pigment. To the best of our knowledge, this is the first report on synthesis 
of gold nanorod using pigment produced by Streptomyces coelicolor by microwave irradiation method. Moreover, the technique has advantageous over physical and chemical synthesis in being rapid, simple and yet green.

\section{Materials and Methods}

\subsection{Materials}

The ingredients of the starch casein agar medium were procured from Hi-media Mumbai, India. The composition of the medium was as follows $(\mathrm{g} / \mathrm{L})$ : Starch $10, \mathrm{~K}_{2} \mathrm{HPO}_{4} 2, \mathrm{KNO}_{3} 2, \mathrm{NaCl} 2, \mathrm{MgSO}_{4} \cdot 7 \mathrm{H}_{2} \mathrm{O}$ $0.05, \mathrm{CaCO}_{3} 0.02, \mathrm{FeSO}_{4} \cdot 7 \mathrm{H}_{2} \mathrm{O} 0.01$, casein 0.3 and agar 15 . The $\mathrm{pH}$ value of the medium is 7.5 . $\mathrm{HAuCl}_{4}$ was also purchased from Hi-media.

\subsection{Identification of Streptomyces coelicolor klmp33 and the pigment}

Streptomyces coelicolor klmp33 isolated from the soil sample of Gulbarga region was identified based on 16S rRNA sequencing and the sequences obtained were submitted to the Gene Bank under the accession No. JQ27722.

The blue pigment produced by Streptomyces coelicolor klmp33 after 3 days of incubation was separated from the biomass by centrifugation the starch casein broth medium at $10000 \mathrm{r} / \mathrm{min}$ for $10 \mathrm{~min}$. The pigment obtained was analyzed using thin layer chromatography conducted on silica-gel $60 \mathrm{~F}_{254}$ plates (Merck) with benzene-acetic acid (9:1, in volume fraction) as solvent. The pigment obtained a single spot with an $R_{\mathrm{f}}$ value of 0.28 indicating the presence of $\operatorname{actinorhodin}^{[13]}$.

\subsection{Synthesis of gold nanorods}

The blue pigment obtained from Streptomyces coelicolor klmp33 was used for the synthesis of gold nanorods. For the synthesis the mixing ratio of $\mathrm{HAuCl}_{4}$ solution to pigment was optimized by interacting different volume of $\mathrm{HAuCl}_{4}$ solution $\left(10^{-3} \mathrm{~mol} / \mathrm{L}\right)$ with different volumes of the pigment and was microwave irradiated for various time periods. Based on the results obtained, the standardized protocol that was followed: $20 \mathrm{~mL}$ of $\mathrm{HAuCl}_{4}$ solution was treated with $1 \mathrm{~mL}$ of the pigment and was microwave irradiated for a period of $120 \mathrm{~s}$.

\subsection{Characterization of gold nanorods}

The synthesis of gold nanorods was preliminary confirmed by UV-visible spectroscopy conducted on Systronics 2200 double-beam UV-visible spectroscopy. The size and shape of the gold nanorods were determined by transmission electron microscopy (TEM, Hitachi H 7500). Samples for this analysis were prepared by coating carbon-coated copper grids with aqueous gold nanoparticles. After $5 \mathrm{~min}$, the extra solution was removed using blotting paper, and then the films on the grids were dried under IR light. The crystalline nature of the gold nanorods was confirmed by X-ray diffraction (XRD, Rigaku Ultima 4 $\mathrm{XRD}$ ). The radiation used was $\mathrm{Cu} K_{\alpha}$ (wavelength is $0.154 \mathrm{~nm}$ ) at $40 \mathrm{kV}$ and $35 \mathrm{~nm}$ with scanning rate of $2^{\circ} / \mathrm{min}$.

To know the probable reducing agent Fourier transform infrared spectroscopy (FTIR) study was carried out. Sample for this analysis was prepared by centrifuging the gold nanoparticles solution at $1 \times 10^{4} \mathrm{r} / \mathrm{min}$ for $20 \mathrm{~min}$. The solid residue was then washed with distilled water to remove any unattached biological moieties from the surface of the nanoparticles. The resultant residue was then dried completely, and the powder was used for FTIR measurements, which was performed on a NICOLET iS5 with Diamond ATR. The FTIR peak was identified and expressed in wave numbers $\left(\mathrm{cm}^{-1}\right)$.

\section{Results and Discussion}

Traditional nanorods were synthesised by seed-mediated synthesis using the surfactant like cetyltrimethylammonium bromide (CTAB), in presence or absence of additives like silver nitrate, but the major drawbacks of these processes are that these processes are multi-stepped ${ }^{[14]}$ and involve toxic chemicals which show immense effect on environment. In contrast, biological syntheses are regarded as nontoxic and alternate way to synthesize gold nanorods. Recently Pariel et al. ${ }^{[15]}$ demonstrated biological synthesis of the gold nanorods using $16 \mathrm{~h}$ biomass of Nostoc ellipsosporum within 48 h. Similarly, gold nanrods synthesis was also reported using Phormidium valderianum biomass in $72 \mathrm{~h}^{[10]}$. In the present study, gold nanorods were synthesized by a easy, safe, rapid and one step bio-based microwave irradiation method in just $120 \mathrm{~s}$ using the pigment produced by Streptomyces coelicolor klmp33.

The main strategy involved in gold nanorods synthesis mainly depends on reduction rate. As reported by Gericke and Pinches, high reduction rate plays a key role in the synthesis of gold nanorods ${ }^{[16]}$. Zhou et al. ${ }^{[17]}$ demonstrated the temperature as important factor which increases the reduction rate for nanoparticles synthesis. Keeping the above points in mind, we used microwave irradiation as thermal factor for the synthesis of the gold nanorods. The microwave radiation heats up a material through its dielectric loss, which converts the radiation energy into thermal energy, which increase rate kinetics of the reaction result in a narrow distribution of the particle ${ }^{[18]}$. The exact mechanism why pigment act as reducing agent is still not clear, to our knowledge it may be soluble nature of the pigment in water which make it one of the best solvent for microwave heating, the pigment act as reductant/ stabilizer in presence of microwave irradiation to assist the conventional solution-phase synthetic strategies based on redox re-actions to prepare gold nanorods ${ }^{[19]}$. 


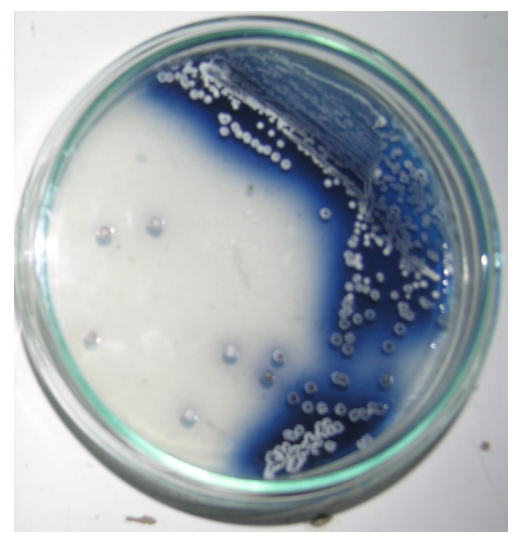

Fig. 1 Streptomyces coelicolor klmp33 on starch casein agar

\subsection{Synthesis of gold nanorods}

Fig. 1 shows the blue pigment produced by Streptomyces coelicolor klmp33, which was used to synthesize gold nanorods from $\mathrm{HAuCl}_{4}$ solution. For the synthesis, $1 \mathrm{~mL}$ of pigment was added to $20 \mathrm{~mL}$ of $\mathrm{HAuCl}_{4}$ solution, and the microwave irradiated. The change in color from light yellow to red wine indicates that the formation of gold nanoparticles ${ }^{[20]}$. The absorbance intensity of the red color light increased steadily as a function of reaction time, as shown in Fig. 2.

\subsection{Characterization of gold nanorods}

The synthesis of gold nanorods was preliminary

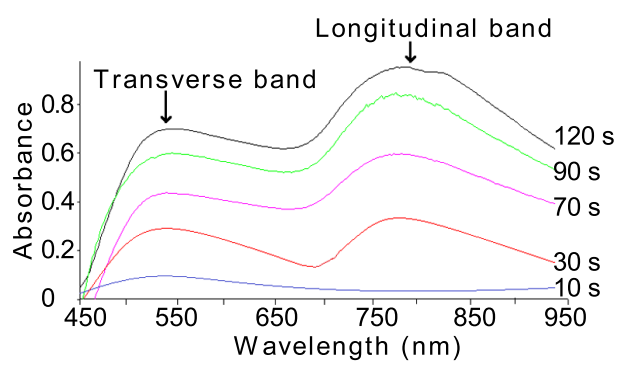

Fig. 2 UV-visible spectrum showing microwave assisted synthesis of gold nanorods recorded as a function of time

confirmed by UV-visible spectroscopy. Fig. 2 shows two peaks, one at visible region and another at nearinfrared region mainly due to transverse and longitudinal surface plasmon resonance respectively, which clearly indicates the synthesis of nanorods ${ }^{[21]}$.

Any changes in the experimental condition can lead to the formation of different shape and size of the nanoparticles $^{[22]}$. So TEM images at different time periods were obtained to see any variation in shape and size of the gold nanoparticles formed. In the initial $10 \mathrm{~s}$ of microwave irradiation, irregular shapes gold nanoparticles having size of about 25-30 nm were formed (Fig. 3(a)). As the reaction time increased up to $30 \mathrm{~s}$ gold naonorods of size $45 \mathrm{~nm}$ were formed (Fig. 3(b)). Similarly gold nanorods were also formed at $90 \mathrm{~s}$ (Fig. 3(c)) and $120 \mathrm{~s}$ (Fig. 3(d)) but the size increased to $200 \mathrm{~nm}$ and $250 \mathrm{~nm}$ respectively.

The synthesis was further confirmed by XRD.

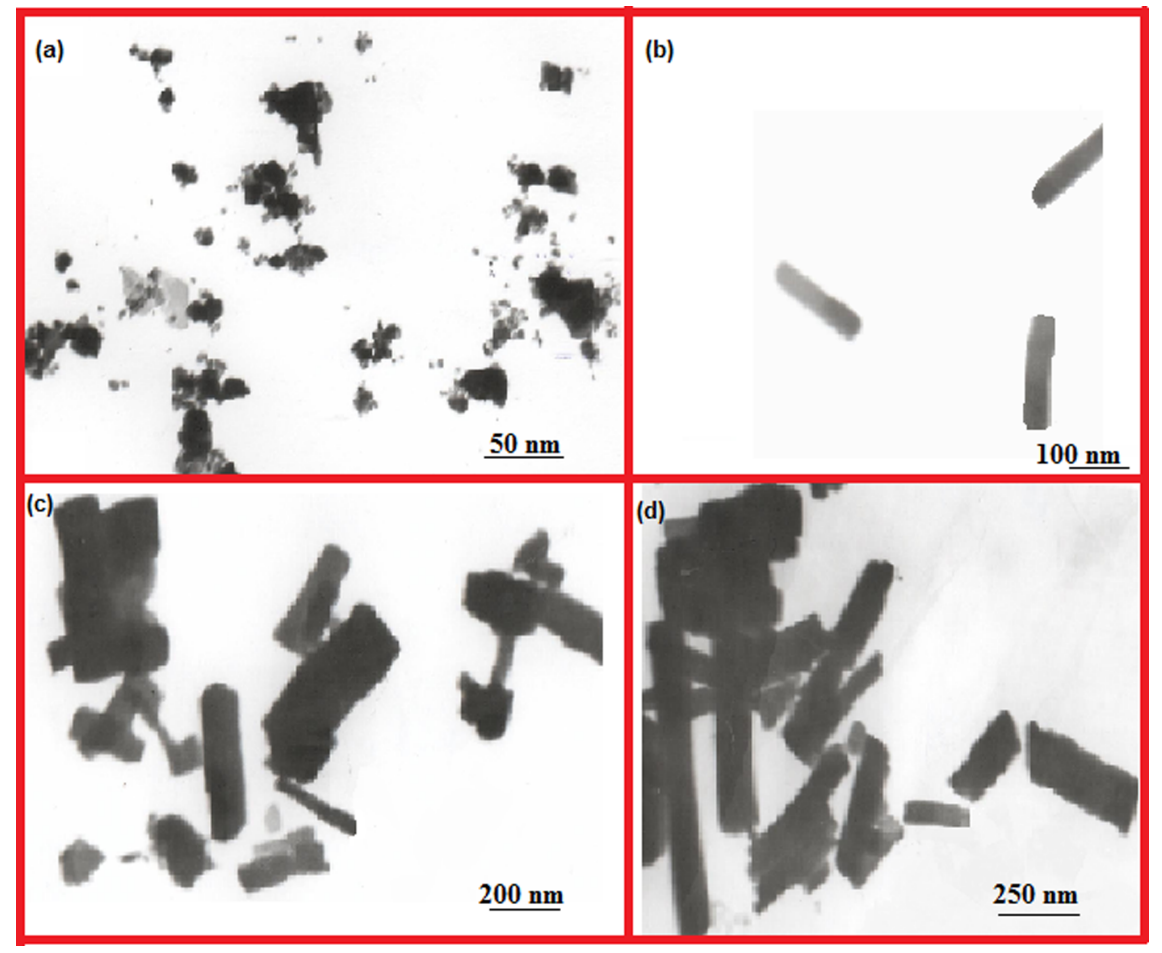

Fig. 3 TEM images of synthesized gold nanoparticles at $10 \mathrm{~s} \mathrm{(a),} 30 \mathrm{~s} \mathrm{(b),} 90 \mathrm{~s}$ (c) and $120 \mathrm{~s}$ (d) 

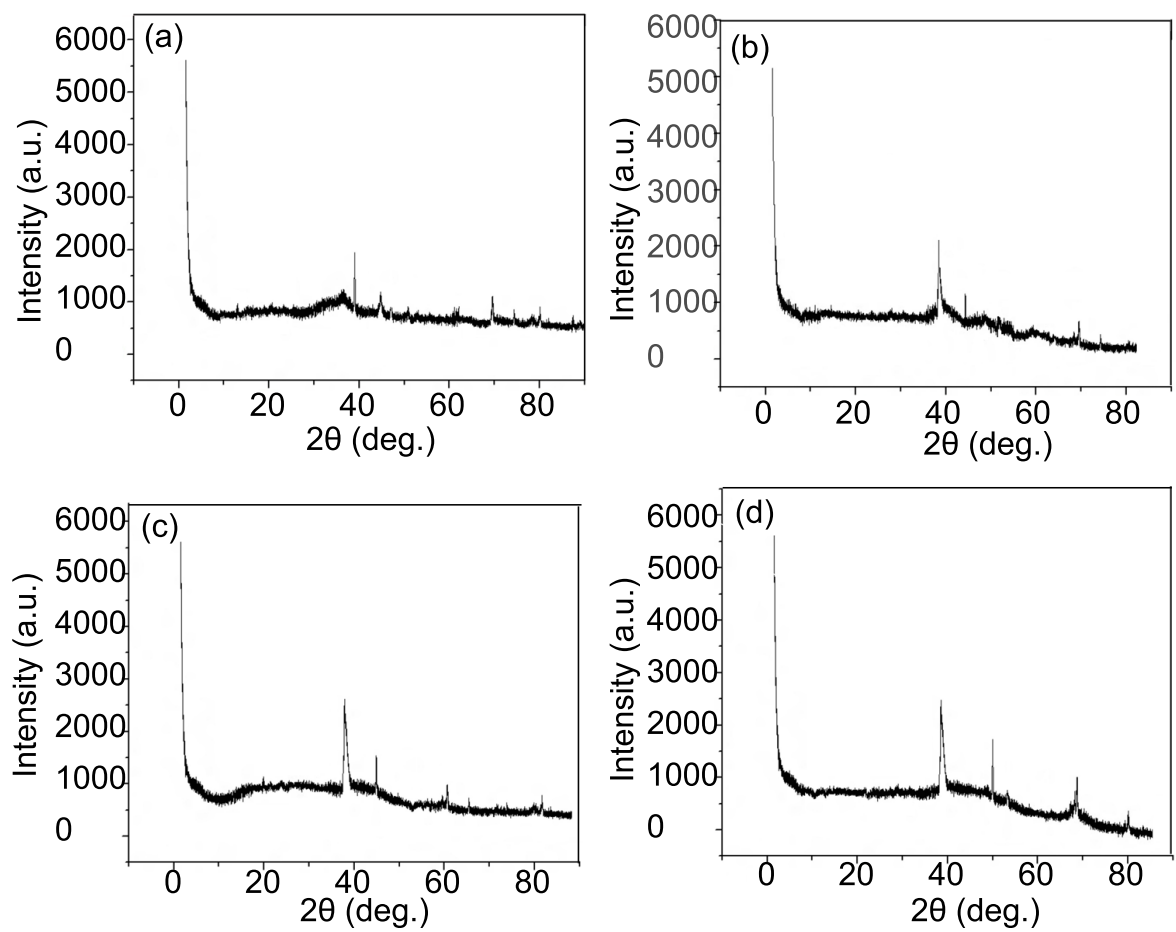

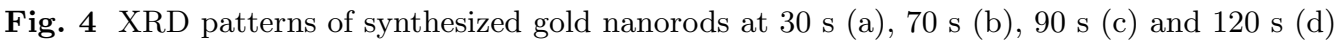
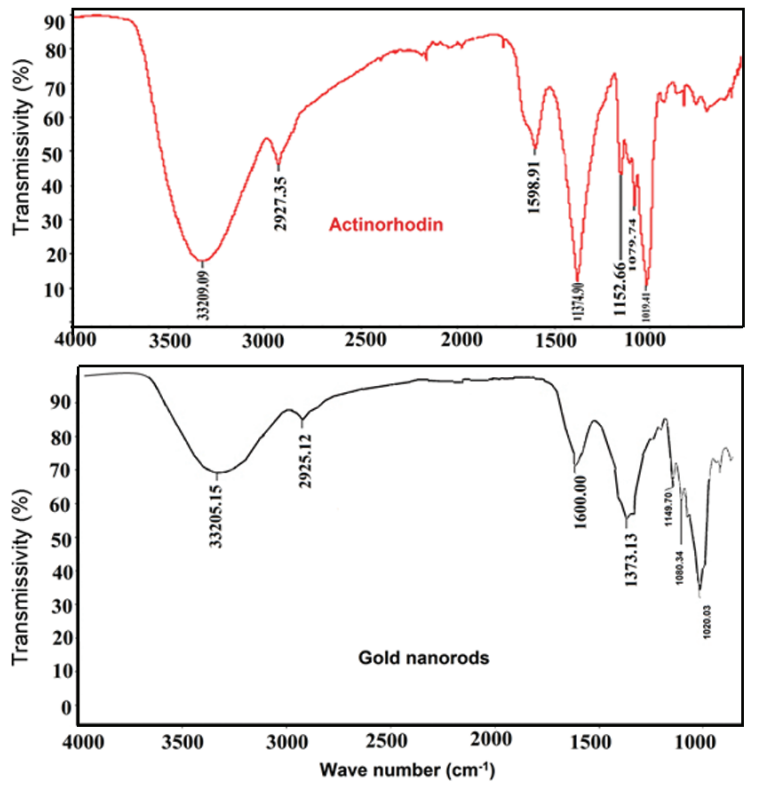

Fig. 5 FTIR spectra of synthesized gold nanorods and actinorhodin

Fig. 4 shows XRD patterns of synthesised gold nanorods in different microwave irradiation time, the four major peaks in all the XRD patterns correspond to the Bragg peaks for the gold nanocrystals were seen. The $2 \theta$ peak values approximately at $39^{\circ}, 44^{\circ}$, $65^{\circ}$ and $77^{\circ}$ correspond to $111,200,220$ and 311 planes of the fcc gold phase respectively.

Fig. 5 shows the FTIR spectra of the purified gold nanorods and pigment. The pigment exhibited absorption peaks at $1152,1598,1645$ and $3320 \mathrm{~cm}^{-1}$ due to cyclic $\mathrm{C}-\mathrm{O}-\mathrm{C}, \mathrm{C}=\mathrm{O}$ and $\mathrm{OH}$ functional groups respectively. The peaks obtained were compared with gold nanorods, less intense peaks with slightly shift were observed in the purified gold nanorods. From the FTIR spectra it may be inferred that pigment was the probable reducing agent which was involved in the synthesis of gold nanorods further the pigment might have formed a layer on the gold nanorods (i.e., biological capping), thus the gold nanorods were stabilized.

\section{Conclusions}

In this study, we report rapid synthesis of gold nanorods in just $120 \mathrm{~s}$ by microwave irradiation method using pigment as a reducing agent. TEM images showed the particles were irregular in shape in the initial $10 \mathrm{~s}$ and changed to rods thereafter. The XRD analysis showed the crystalline nature of gold nanorods. Further, FTIR studies showed pigment as probable reducing agent responsible for the synthesis of gold nanorods. The synthesis developed in this study has distinct advantageous over biological and chemical methods in being rapid, safe, non-toxic and further, the synthesis of gold nanorods by this route is the appropriate way to develop green technology for large scale synthesis of gold nanorods.

\section{Acknowledgements}

Authors acknowledge Prof. A. Venktaraman, Chairman, Department of Materials Science, Gulbarga Univer- 
sity, Gulbarga for providing FTIR facility.

\section{REFERENCES}

[1] T. Pradeep and Anshup, Thin Solid Films. 517 (2009) 6441.

[2] K. Kalishwaralal, V. Deepak, S.B.R. Kumar Pandian, M. Kottaisamy, S. BarathManiKanth, B. Kartikeyan and S. Gurunathan, Colloids Surf. B 77 (2010) 262.

[3] E. Filippo, A. Serra, A. Buccolieri and D. Manno, J. Non-Cryst. Solids 356 (2010) 350.

[4] E. Castro-Longoria, A.R. Vilchis-Nestor and M. Avalos-Borja, Colloids Surf. B 83 (2011) 48.

[5] H. Bar, D.K. Bhui, G.P. Sahoo, P. Sarkar, S. Pyne, D. Chattopadhyay and A. Misra, J. Exp. Nanosci. 7 (2012) 119.

[6] F.K. Derakhshan, A. Dehnad and M. Salouti, Synth. React. Inorg., Met.-Org., Nano-Met. Chem. 42 (2012) 871.

[7] D. Pissuwan, S.M. Valenzuela and M.B. Cortie, Biotechnol. Genet. Eng. Rev. 25 (2008) 112.

[8] J.C. Zhou, C.M. Soto, M.S. Chen, M.A. Bruckman, M.H. Moore, E. Barry, B.R. Ratna, P.E. Pehrsson, B.R. Spies and T.S. Confer, J. Nanobiotechnol. 10 (2012) 12

[9] T.S. Hauck, T.L. Jennings, T. Yatsenko, J.C. Ku- maradas and W.C.W. Chan, Adv. Mater. 20 (2008) 3838.

[10] D. Parial, H.K. Patra, A.Kr. Dasgupta and R. Pal, Eur. J. Phycol. 47 (2012) 29.

[11] B.M. Stacy, Ph.D Thesis, University of Dayton, 2012.

[12] D. Manikprabhu and K. Lingappa, Saudi. J. Biol. Sci. 20 (2013) 168.

[13] D. Manikprabhu and K. Lingappa, J. Pharmy. Res. 6 (2013) 260

[14] R.W. Bourne, S.M. Kelly and J.D. Wadhawan, J. Exp. Nanosci. 7 (2012) 687.

[15] D. Parial, H.K. Patra, P. Roychoudhury, A.K. Dasgupta and R. Pal, J. Appl. Phycol. 24 (2012) 60.

[16] M. Gericke and A. Pinches, Hydrometallurgy 83 (2006) 140

[17] Y. Zhou, H. Itoh, T. Uemura, K. Naka and Y. Chujo, Langmuir 18 (2002) 283.

[18] N. Saifuddin, C.W. Wong and A.A. Nur Yasumira, E-J. Chem. 6 (2009) 70.

[19] S.P. Zhug, S.C. Tang and X.K. Meng, Chin. Phys. Lett. 26 (2009) 078104.

[20] J.C. Mohan, G. Praveen, K.P. Chennazhi, R. Jayakumar and S.V. Nair, J. Exp. Nanosci. 8 (2013) 45.

[21] A. Martin, C. Schopf, A. Pescaglini, A.O. Riordan and D. Lacopino, J. Exp. Nanosci. 7 (2012) 702.

[22] X. Huang, S. Neretina and M.A. El-Sayed, Adv. Mater. 21 (2009) 4910. 